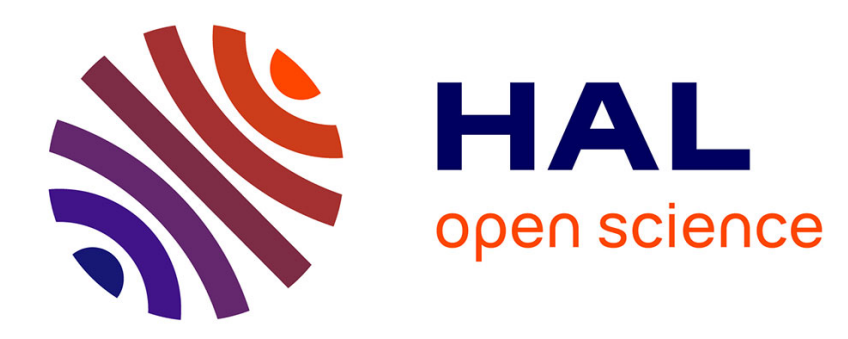

\title{
La basilique Saint-Appien de Sagone (Vico, Corse-du-Sud)
}

Daniel Istria

\section{To cite this version:}

Daniel Istria. La basilique Saint-Appien de Sagone (Vico, Corse-du-Sud). Mélanges de l'École française de Rome - Moyen Âge, 2021, 133-1, pp.121-130. 10.4000/mefrm.8523 . hal-03453785

\section{HAL Id: hal-03453785 \\ https://hal.science/hal-03453785}

Submitted on 7 Dec 2021

HAL is a multi-disciplinary open access archive for the deposit and dissemination of scientific research documents, whether they are published or not. The documents may come from teaching and research institutions in France or abroad, or from public or private research centers.
L'archive ouverte pluridisciplinaire $\mathbf{H A L}$, est destinée au dépôt et à la diffusion de documents scientifiques de niveau recherche, publiés ou non, émanant des établissements d'enseignement et de recherche français ou étrangers, des laboratoires publics ou privés. 
Mélanges

de l'École française de Rome

Moyen Âge

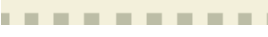

\section{Mélanges de l'École française de Rome - Moyen Âge}

133-1 | 2021

Reimpiego, rilavorazione, rifunzionalizzazione: la "lunga vita" della scultura medievale nei cantieri di Età moderna - Varia

\section{La basilique Saint-Appien de Sagone (Vico, Corse- du-Sud)}

Nouvelles données et nouvelles interprétations

Daniel Istria

\section{(2) OpenEdition}

\section{Édition électronique}

URL : https://journals.openedition.org/mefrm/8523

DOI : $10.4000 /$ mefrm. 8523

ISSN : $1724-2150$

Éditeur

École française de Rome

\section{Édition imprimée}

Date de publication : 1 juin 2021

Pagination : 121-130

ISBN : 978-2-7283-1487-4

ISSN : 1123-9883

Ce document vous est offert par Centre national de la recherche scientifique (CNRS)

\section{cnrs}

\section{Référence électronique}

Daniel Istria, « La basilique Saint-Appien de Sagone (Vico, Corse-du-Sud) », Mélanges de l'École française de Rome - Moyen Âge [En ligne], 133-1 | 2021, mis en ligne le 01 juin 2021, consulté le 07 décembre 2021. URL : http://journals.openedition.org/mefrm/8523 ; DOI : https://doi.org/10.4000/ mefrm. 8523 


\title{
La basilique Saint-Appien de Sagone (Vico, Corse-du-Sud)
}

\author{
Nouvelles données et nouvelles interprétations
}

\author{
Daniel Istria
}

CNRS, Aix Ma rseille Université, LA3M, Aix-en-Provence - Daniel ISTRIA distria@mmsh.univ-aix.fr

\begin{abstract}
Découverte au début des années 1960, la basilique paléochrétienne de l'agglomération de Sagone, dans le sud-ouest de la Corse, a fait l'objet de plusieurs interprétations, parfois contradictoires. La reprise des recherches archéologiques entre 2006 et 2017 ont permis de collectées des informations inédites qui renouvellent complètement la connaissance de ce complexe. Seules les questions relatives à la chronologie, l'architecture, les aménagements liturgiques et la titulature du premier état de l'édifice sont abordées dans cet article. Les nouvelles données invitent à une réflexion plus large sur la première Église de Corse et le développement du culte des saints.
\end{abstract}

Corse, basilique, architecture, aménagement liturgique, culte des saints

Discovered at the beginning of the 1960s, the paleochristian basilica of the town of Sagone, in the southwest of Corsica, has been the object of several interpretations, sometimes contradictory. The resumption of archaeological research between 2006 and 2017 has allowed the collection of new information that completely renews our knowledge of this church. Only questions relating to the chronology, the architecture, the liturgical arrangements and the title of the first state of the edifice are addressed in this article. The new data invites a wider reflection on the first Church of Corsica and the development of the cult of the saints.

Corsica, basilica, architecture, liturgical installation, worship of the saints

C'est en 1964 puis en 1978 que furent découverts sous la cathédrale médiévale de Sagone (XII ${ }^{\mathrm{e}}$ siècle) les vestiges censés appartenir à deux édifices de culte chrétien successifs' ${ }^{1}$. La construction

1. L'agglomération de Sagone (commune de Vico) est située sur le littoral sud-occidental de la Corse, à une trentaine de kilomètres au nord d'Ajaccio. L'évêché de Sagone Saone est mentionné pour la première fois en 591 (Gregorii I papae registrum epistolarum, lib. I, ep. 76 et 79, août 591). La construction d'un baptistère au sud de l'église durant la seconde moitié ou plus probablement le dernier tiers du VI ${ }^{e}$ siècle, laisse penser qu'il n'est institué qu'à ce moment là (Istria - Françoise - Pellegrino, 2012). Après avoir été supprimé vers la fin du VII ${ }^{e}$ siècle il est réactivé à la fin du XI ${ }^{e}$ siècle et sa cathédrale reconstruite quelques années plus tard. En 1572, le siège est déplacé à Vico, une agglomération distante d'une vingtaine de kilomètres, puis au la plus ancienne fut interprétée par G. MoracchiniMazel comme un baptistère de plan cruciforme appartenant au groupe épiscopal et daté du $\mathrm{IV}^{\mathrm{e}}$ siècle, peut-être «de la décennie 370 ». Durant une seconde phase, datée du $\mathrm{V}^{\mathrm{e}}$ siècle, cet édifice aurait été remplacé par une grande basilique, peutêtre à cinq nefs, terminée à l'est par une abside semi-circulaire intérieurement et polygonale à trois pans à l'extérieur².

Dans les années 1990, N. Duval en fit une relecture rapide sur la base de la documentation

XVII ${ }^{e}$ siècle à Calvi. Le diocèse est définitivement supprimé en 1801.

2. Moracchini-Mazel 1966, 1972, 1988 et 2004. 
produite par la responsable des fouilles. Il accepta sans réserve l'identification de deux états consécutifs et n'écarta pas une datation autour de la seconde moitié du IV siècle pour le plus ancien. Concernant l'état II, l'existence d'un premier mur transversal au nord et d'un second à l'ouest ne permettrait pas selon lui de reconnaître un plan basilical classique, mais il ne proposa pas de nouvelle interprétation. Une datation «un peu plus tardive [que le $\mathrm{V}^{\mathrm{e}}$ siècle]" lui semblait possible ${ }^{3}$.

Sans pourtant disposer d'informations nouvelles Ph. Pergola et F. Di Renzo restituent quant à eux une basilique à trois nefs. Sa fondation devrait se situer vers la fin du $V^{\mathrm{e}}$ ou le début du VI ${ }^{\mathrm{e}}$ siècle par comparaison avec la basilique intra-muros de Mariana ${ }^{4}$.

L'ensemble de ces vestiges a été réexaminé entre 2006 et $2017^{5}$. Les murs encore visibles ont fait l'objet d'une relecture attentive et des fouilles ont été réalisées autour et à l'intérieur de l'édifice médiéval. Les données collectées renouvellent complètement la connaissance de ce complexe ${ }^{6}$.

L’hypothèse de la présence de deux édifices de culte peut être écartée aujourd'hui. Les vestiges du "premier état», mais aussi une partie de ceux du "deuxième état», ont été confondus avec des éléments appartenant à une habitation construite au début du IV siècle. Il n'existe donc qu'une basilique antérieure à la cathédrale romane du XII ${ }^{e}$ siècle et seuls ses aménagements liturgiques ont été transformés durant le premier Moyen Âge. On ne présentera ici que l'état initial de cet édifice, son architecture, sa chronologie ainsi que sa titulature.

3. Duval 1995.

4. Pergola - Di Renzo 2001, p. 116-117. On sait toutefois aujourd'hui que la basilique intra-muros de Mariana, que l'on peut identifier comme la cathédrale, est construite au tout début du Ve siècle (Istria - Dixneuf - Françoise, 2014).

5. Cette recherche a été conduite sous la direction de D. Istria. Outre l'étude du bâti, les fouilles ont été étendues dans l'église ainsi qu'au nord et au sud de celle-ci sur une superficie de $520 \mathrm{~m}^{2}$.

6. On trouvera plus de détails et des précisions sur l'historique des recherches, l'extension des fouilles et les édifices postérieurs à la basilique paléochrétienne dans : Istria 2011 et 2014.

\section{LA BASILIQUE ET SES AMÉNAGEMENTS LITURGIQUES}

La basilique a été installée à l'intérieur d'une habitation antérieure qui semble avoir été arasée à la hauteur de ses fondations (fig. 1).

L'édifice chrétien est constitué d'une nef unique d'une largeur dans l'œuvre de 7,7 m (fig. 2). Sa longueur totale n'est pas précisément connue, mais elle ne peut pas dépasser $20 \mathrm{~m}$ en raison de la présence d'un autre bâtiment à l'ouest. Vers l'est la nef est terminée par une abside polygonale à trois pans à l'extérieur et semi-circulaire à l'intérieur de 4,80 $\mathrm{m}$ d'ouverture et 2,40 $\mathrm{m}$ de profondeur.

Tous les murs, d'une épaisseur de 70 à $80 \mathrm{~cm}$, sont caractérisés par l'utilisation d'un appareil mixtum de bonne qualité. Les assises de moellons de granite, de forme et de dimensions hétérogènes (jusqu'à $40 \mathrm{~cm}$ de longueur), alternent de manière irrégulière avec des assises composées de fragments de terre cuite architecturale, de panse d'amphore et de fines pierres plates. Le mortier à base de chaux et de sable est abondant et très résistant.

Les pierres de construction semblent prélevées sur le site même. Il peut s'agir de moellons remployés provenant du démontage des murs de l'habitation antérieure, mais aussi du débitage des affleurements rocheux locaux. Le sable utilisé dans la préparation du mortier pourrait être prélevé sur la rive toute proche du fleuve Sagone, distante actuellement de seulement $160 \mathrm{~m}$. Quant à la chaux, elle peut avoir été produite avec des roches présentes dans la vallée. Cependant, des blocs de calcaire de Bonifacio, dont le gisement accessible par la mer est distant d'environ $120 \mathrm{~km}$, ont été retrouvés sur le site dans les contextes contemporains de la construction. On a ainsi pu privilégier ce matériau bien mieux adapté à la fabrication de la chaux que les ressources locales.

Il n'a pas été retrouvé de niveau pouvant être clairement identifié comme résultant de l'effondrement de la toiture de la basilique. Néanmoins, parmi les très nombreux exemplaires de tegulae rencontrés autour de l'édifice certains sont marqués d'estampilles explicitement chrétiennes dont le texte permet, sans grand risque d'erreur, de penser qu'elles étaient destinées à couvrir la basilique ( $c f$. infra). La nature de l'argile utilisée pour leur confection et surtout la découverte à quelques mètres de l'édifice de culte d'un four de 


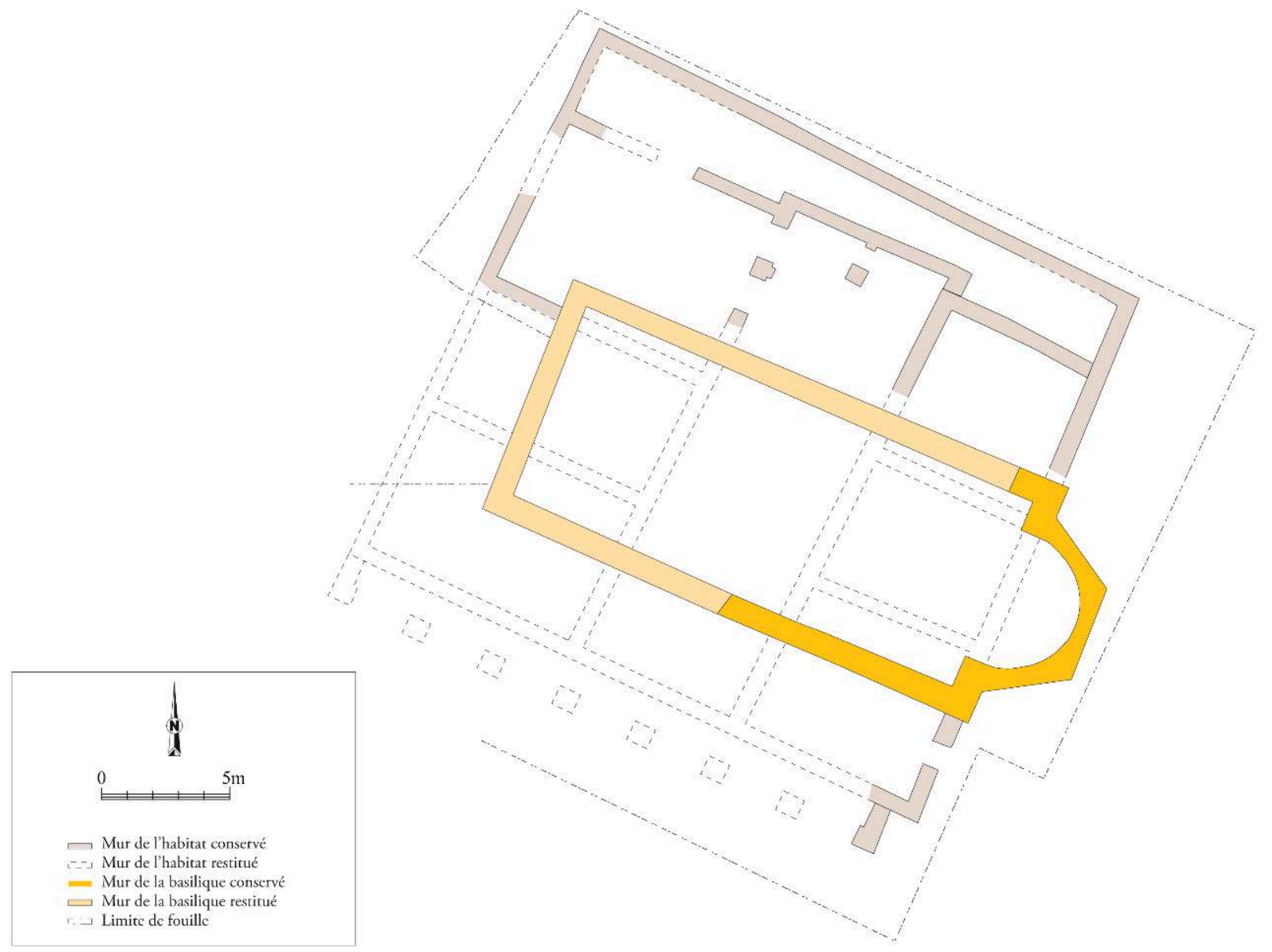

Fig. 1 - Plan général de la basilique et des vestiges de l'habitation antérieure.

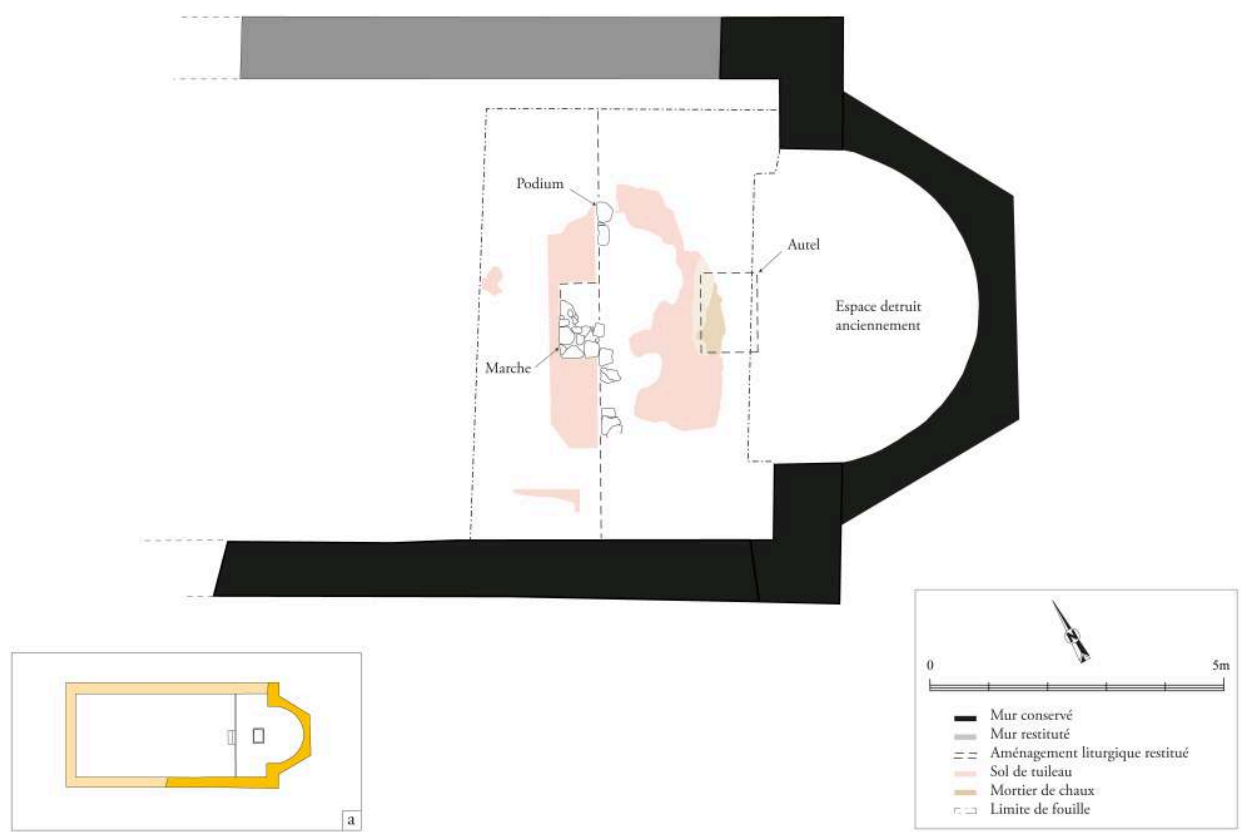

Fig. 2 - Plan des vestiges du premier état de la basilique et proposition de restitution (a). 
chronologie semblable ne laissent planer aucun doute sur le lieu de production de ces matériaux de couverture.

La nef disposait d'un sol de mortier de chaux contenant du gravier et de nombreux fragments de terre cuite de petites dimensions ${ }^{7}$. À son extrémité orientale un emmarchement axial, dont la trace de l'arrachement a été conservée dans le sol $^{8}$, permettait d'accéder à un presbyterium surélevé de 31 à $41 \mathrm{~cm}^{9}$. Cette estrade occupait toute la largeur de l'édifice sur une profondeur de 2,97 m (fig. 3). Elle se prolongeait très probablement dans l'abside dont le sol n'est plus visible aujourd'hui ${ }^{10}$. Une série d'empreintes conservées sur sa face occidentale trahit sans doute la présence d'un placage de pierre d'environ 1,5 cm d'épaisseur, anciennement arraché. Le sol est formé, comme celui de la nef, d'un terrazzo de 8 à $9 \mathrm{~cm}$ d'épaisseur. Relativement horizontal (altitude sup. : 10,52 à 10,55 m NGF), il est soigneusement lissé en surface mais comporte de nombreuses lacunes résultant de creusements.

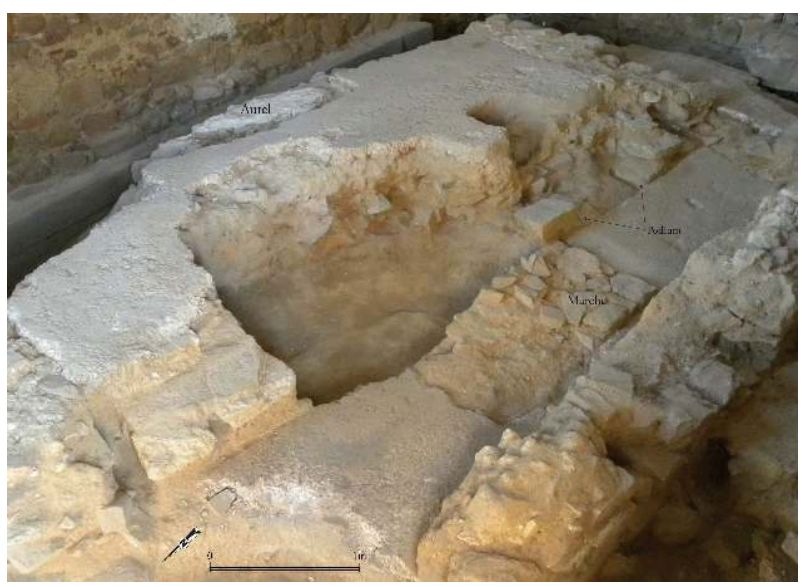

Fig. 3 - Le presbyterium du premier état de la basilique.

7. Jusqu'à environ $2 \mathrm{~cm}$ de côté. Un sol identique a été retrouvé dans la nef de la basilique intra-muros de Mariana.

8. Cette empreinte est de forme rectangulaire de $1,27 \mathrm{~m}$ (nord-sud) × 0,64 m (est-ouest). À l'intérieur se trouvaient des petits moellons de granite, majoritairement plats et soigneusement agencés. Ils n'étaient toutefois conservés que dans la moitié sud sur une hauteur de $10 \mathrm{~cm}$.

9. Compte tenu de la hauteur de l'estrade cet escalier pouvait compter deux marches.

10. Le sol de l'abside a été entièrement fouillé en 1964 et non documenté. Sans doute avait-il déjà été fortement endommagé lors de la construction de l'abside romane au XII ${ }^{e}$ siècle. De fait, il est impossible de connaître sa nature et sa hauteur. On peut toutefois raisonnablement penser qu'il était à la même hauteur ou plus haut que le presbyterium situé en avant.
En revanche, il est peu usé et ne présente pas de trace de restauration ancienne.

À sa surface une importante lacune est visible au niveau de l'axe médian est-ouest et à $80 \mathrm{~cm}$ en avant de la corde de l'abside. Cette trace se différencie des autres lacunes par sa relative régularité et son plan subrectangulaire de 1,54 m de longueur nord-sud et d'au moins $50 \mathrm{~cm}$ dans le sens est-ouest ${ }^{11}$. Au centre est visible une large plaque de mortier de chaux bien plus fin que celui utilisé pour la confection du sol et dépourvu de gravier et de fragments de terre cuite. Sa surface régulière mais non lissée se situe à $5 \mathrm{~cm}$ sous le niveau du sol. L'observation des sections ne permet pas de repérer de mortier identique en d'autres points de l'estrade. Par conséquent, plutôt qu'une simple lacune résultant d'un creusement intempestif, il semblerait qu'il s'agisse là de la trace ou plus exactement de l'arrachement d'un élément de plan rectangulaire ou carré. Compte tenu de sa position et de ses dimensions mais surtout des aménagements retrouvés exactement au-dessus et appartenant donc aux phases successives ${ }^{12}$, il est possible de l'interpréter comme l'empreinte du socle d'un autel qui pouvait être scellé par le mortier de chaux retrouvé au centre. Probablement faut-il restituer ici une table dont un fragment en marbre blanc a été retrouvé hors contexte au nord de l'édifice (fig. 4) ${ }^{13}$. On peut aussi lui associer deux tronçons de colonnettes, également en marbre blanc, de 10,2 cm et $9,5 \mathrm{~cm}$ de diamètre, provenant du comblement de sépultures médiévales.

\section{LA DATATION DE LA BASILIQUE}

Un sondage réalisé à l'extérieur et à l'est de la basilique a permis de mettre en évidence la tranchée de fondation de l'abside. Elle était comblée de fragments de céramique, dont certains de grande

11. Le côté oriental a été détruit très probablement par la tranchée réalisée dans les années 1960 ou bien lors de la construction du mur oriental de la chapelle en 1728.

12. Les traces de trois autels successifs (seconde moitié $\mathrm{V}^{\mathrm{e}}, \mathrm{XII}{ }^{\mathrm{e}}$ et XVIII ${ }^{\text {e }}$ siècle) ont été reconnues dans les niveaux supérieurs.

13. Fragment de marbre blanc de très belle qualité. Dimensions conservées : $27 \times 17,5 \mathrm{~cm}$. Bord de $6,5 \mathrm{~cm}$ d'épaisseur avec listel sur la partie supérieure de $8,2 \mathrm{~cm}$ de largeur décoré de deux rainures rectilignes et cuvette centrale de $2 \mathrm{~cm}$ de profondeur avec léger ressaut. 

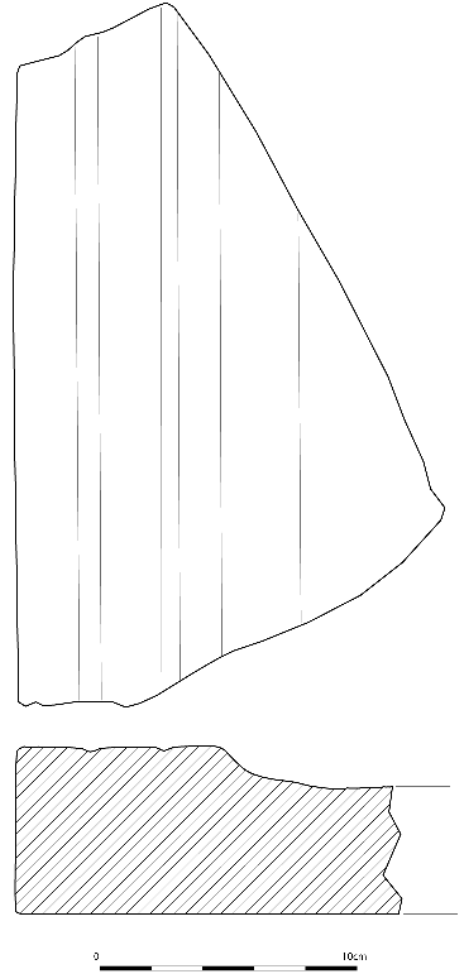

Fig. 4 - Dessin du fragment de table d'autel en marbre.

dimension (jusqu'à $30 \mathrm{~cm}$ de longueur), destinés à assurer le drainage de ce secteur. On présente ci-dessous la liste des fragments identifiés parmi les 200 prélevés $^{14}$ (fig. 5).

Tab. 1 - Inventaire des céramiques identifiées découvertes dans la tranchée de fondation de l'abside de la basilique de Sagone (identification E. Pellegrino).

\begin{tabular}{|c|c|c|c|}
\hline $\mathrm{N}^{\circ}$ dessin & Catégorie & Type & Datation \\
\hline & Amphore italique & Empoli & $200-450$ \\
\hline 1 & Amphore africaine & Tripolitaine III & $150-400$ \\
\hline 2 & Amphore africaine & Keay 25.1 & IV $^{e}$ siècle \\
\hline 3 & Sigillée claire D & Hayes 59 & $320-420$ \\
\hline 4 & Africaine de cuisine & Hayes 23B & $100-400$ \\
\hline 5 & Africaine de cuisine & Hayes 23B & $100-400$ \\
\hline 6 & Africaine de cuisine & Hayes 197 & $100-400$ \\
\hline 7 & Africaine de cuisine & Hayes 181D & $350-450$ \\
\hline 8 & Africaine de cuisine & Hayes $182 \mathrm{C}$ & Fin $\mathrm{II}^{\mathrm{e}}$-III ${ }^{\mathrm{e}}$ siècle \\
\hline 9 & Africaine de cuisine & $\begin{array}{l}\text { Bonifay culinaire } \\
\text { (C/A) type } 13\end{array}$ & $\begin{array}{l}\text { Fin } \mathrm{IV}^{\mathrm{e}} \text {-première } \\
\text { moitié } \mathrm{V}^{\mathrm{e}} \text { siècle }\end{array}$ \\
\hline 10 & Africaine de cuisine & Hayes 196 & $\begin{array}{l}\mathrm{III}^{\mathrm{e}} \text {-début } \\
\mathrm{V}^{\mathrm{e}} \text { siècle }\end{array}$ \\
\hline
\end{tabular}

14. L'étude de la céramique a été conduite par E. Pellegrino.
Dans le but de préciser la datation cinq petits échantillons de charbon de bois prélevés à l'intérieur du mortier de chaux du sol du presbyterium ont fait l'objet d'une analyse radiocarbone (AMS). Il est très probable qu'il s'agisse du bois utilisé pour la calcination de la pierre lors de la fabrication de la chaux. Les dates obtenues sont les suivantes :

\footnotetext{
PR5 : Beta - 355313, date calibrée 2 sigma (95\% de probabilité) : 320 à $420 \mathrm{AD}$

PR6 : Beta - 355314, date calibrée 2 sigma (95\% de probabilité) : 410 à $550 \mathrm{AD}$

PR7 : Beta - 471547, date calibrée 2 sigma (95\% de probabilité) : 248 à $390 \mathrm{AD}$

PR8 : Beta - 471548, date calibrée 2 sigma (95\% de probabilité) : 382 à $538 \mathrm{AD}$

PR9 : Beta - 471549, date calibrée 2 sigma (95\% de probabilité) : 346 à $536 \mathrm{AD}$
}

Les céramiques découvertes dans la tranchée de fondation de l'abside sont toutes antérieures au milieu du $\mathrm{V}^{\mathrm{e}}$ siècle. Plusieurs productions s'arrêtent cependant vers 400 (amphores Tripolitaine III et Keay 25.1, céramique de cuisine Hayes 23B et 197). Les plus récentes ne peuvent être antérieures à la fin du $\mathrm{IV}^{\mathrm{e}}$ siècle (Bonifay culinaire (C/A) type 13). Quant aux datations par le radiocarbone, elles couvrent une large plage chronologique (248-550) mais se recoupent vers la fin du $\mathrm{IV}^{\mathrm{e}}$ et le début $V^{e}$ siècle. L'échantillon PR6 ne peut toutefois pas être antérieur à 410 .

Une fourchette chronologique entre 410 et 450 environ peut donc être retenue même si, compte tenu des données céramologiques, les premières décennies du $\mathrm{V}^{\mathrm{e}}$ siècle semblent mieux convenir (fig. 6).

\section{LA TITULATURE}

On sait par les textes modernes que la cathédrale de Sagone était alors dédiée à saint Appien. Le plus ancien document conservé est le procèsverbal de la visite apostolique de la Corse faite en 1686 par Mgr Giovanni Battista Spinola, évêque de Luni et Sarzana. Il indique l'existence à Sagone "d'un édifice ruiné... qui avait servi d'église cathédrale de ce diocèse, sous la titulature de saint 


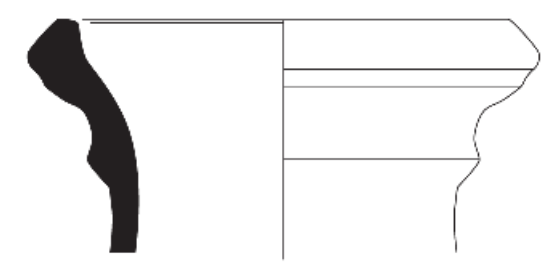

1

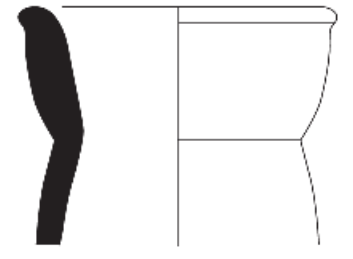

2

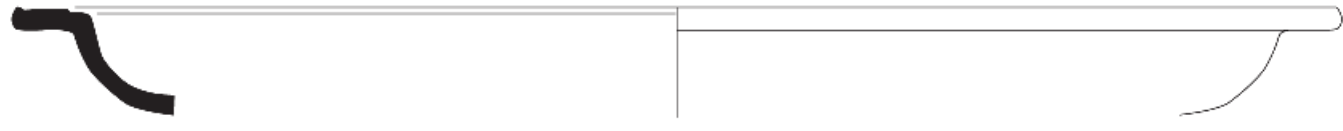

3

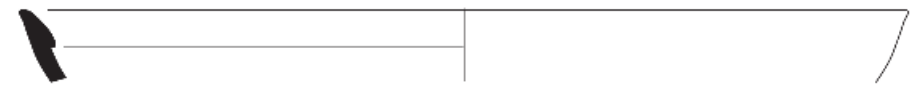

4

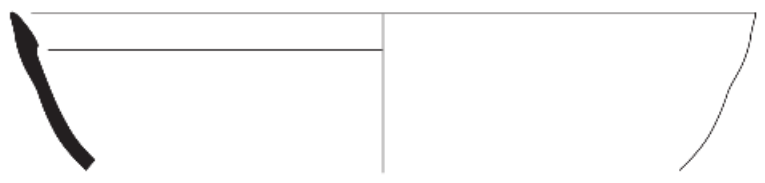

5

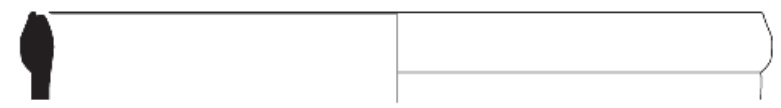

6

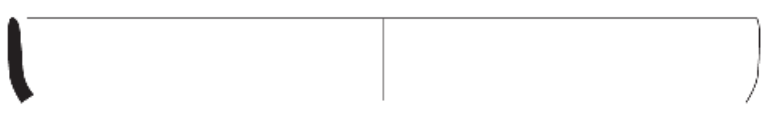

7

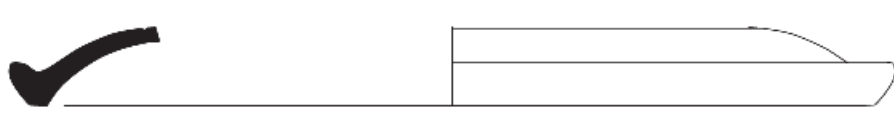

8

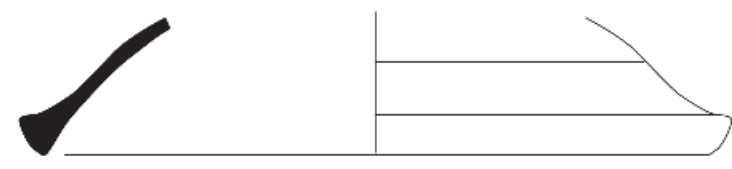

9

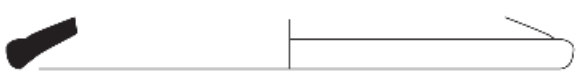

10

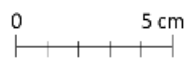

Fig. 5 - Céramiques découvertes dans la tranchée de fondation de l'abside de la basilique (étude et dessins E. Pellegrino).

Appien ${ }^{15}$. Ce patronage est confirmé en 1728 par

15. Rome, Biblioteca Casanatense, Mss varia, n. 204 : Ibi fuere ei obviam Domini Canonici et aliae ecclesiasticae personae sagonensis dioecesis eos comitatus vespere prosequens terrestre iter invenit prope mare aedificium diructum, et in eo adhuc remanet a quatuor lateribus parum a terra elevatis parietes, quod ut fuit dictum inserviebat pro cathedrali ecclesia dictae dioecesis sub titulo Sancti Apiani. Transcription et traduction de L. Belgodere. 


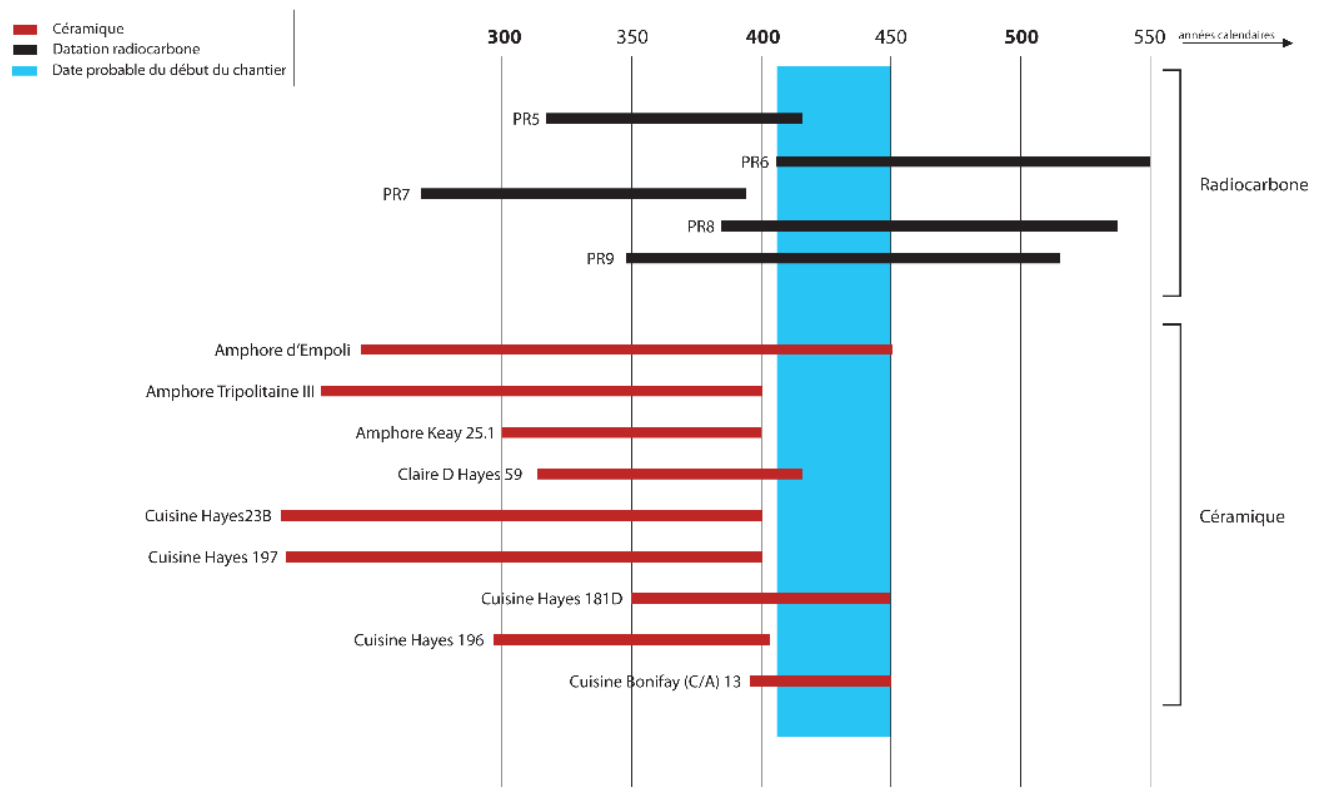

Fig. 6 - Synthèse chronologique de la basilique.
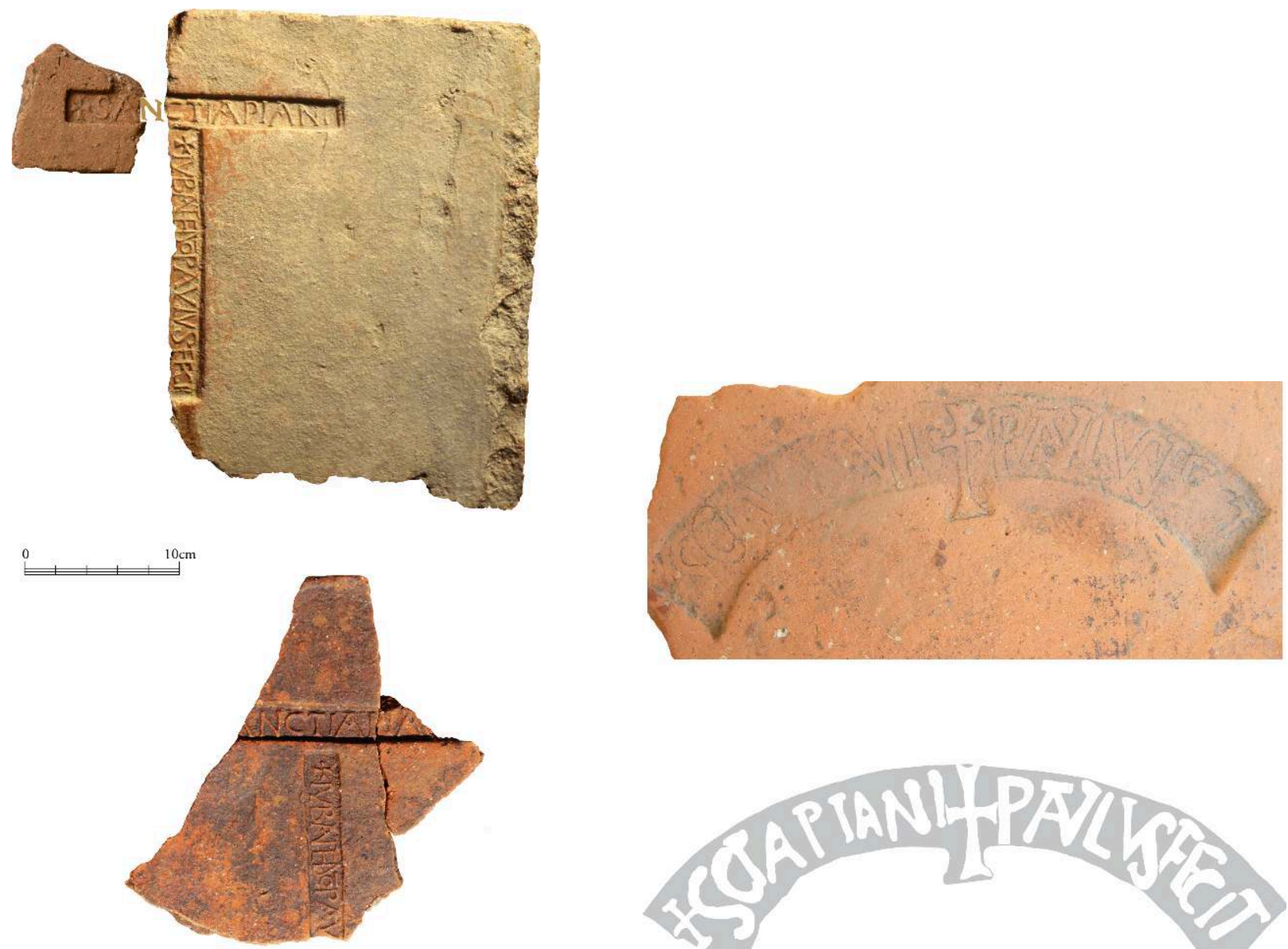

Fig. 7 - Estampille sur tuile de type 1 (fragment de droite découvert par G. Moracchini-Mazel, photo C. Andreani).

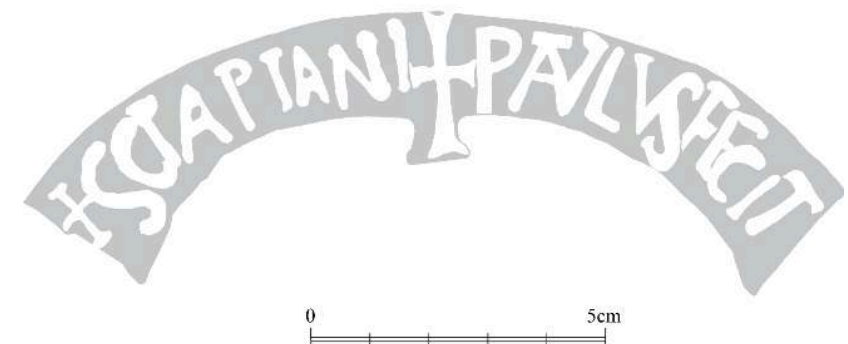

Fig. 8 - Estampille sur tuile de type 2. 
le procès-verbal d'une nouvelle consécration de l'édifice suite à sa reconstruction partielle ${ }^{16}$.

La documentation médiévale n'apporte aucune information à ce sujet. En revanche, des estampilles sur tuiles attestent de l'ancienneté de cette dédicace à saint Appien.

$\mathrm{Au}$ total huit timbres ont été découverts durant les fouilles autour de la basilique dans divers contextes dont les plus anciens sont datés par la céramique de la première moitié ou du milieu du $\mathrm{V}^{\mathrm{e}}$ siècle $^{17}$. Ils sont donc contemporains du premier état de l'édifice de culte même si l'on ne peut affirmer de manière catégorique que la basilique était bien placée sous la protection d'Appien dès sa fondation.

Deux types de timbres ont été identifiés et ont pu être entièrement reconstitués.

Le premier (type 1) (fig. 7), dont cinq exemplaires ont été retrouvés, est constitué de deux cartouches rectangulaires correspondant à deux matrices différentes mais assemblées pour former un tau. L'un et l'autre ont une longueur de $17,8 \mathrm{~cm}$ et une largeur de $2,2 \mathrm{~cm}$. Chacun porte une inscription :

\section{+SANCTI APIANI / + IVBANTE D(E)O PAVLVS \\ FECIT}

Le second timbre (type 2), connu en trois exemplaires, est un cartouche en arc de cercle de 2,2 cm de largeur aux extrémités et $1,8 \mathrm{~cm}$ au centre, pour $14,3 \mathrm{~cm}$ de longueur (fig. 8). La partie centrale est occupée par une croix dont le bras inférieur dépasse de $1 \mathrm{~cm}$ du cartouche dans la partie concave. Le texte occupe l'ensemble du cartouche :

\section{+SCI APIANI+PAVLVS FECIT}

Étant donné que la forme des lettres est très proche et que ces deux timbres ont été trouvés dans des contextes contemporains, il est très probable que ce Paul ne soit qu'un seul et même personnage. De même, tout pousse à croire qu'il s'agit du même Paul, évêque de Corse, mentionné sur

16. Archives départementales de Corse du Sud, Ajaccio, 4G47/17, 1728.

17. Un premier fragment du timbre avait été découvert en 1965 : Moracchini-Mazel 1966. une autre estampille avec monogramme en forme de croix avec les lettres ECLS entouré de l'inscription PA[V]LVS EP[I]SCOPVS CORSIC[AE], découvert lui aussi à Sagone dans un contexte également du milieu du $V^{e}$ siècle $^{18}$. Ce Paul pourrait donc être l'évêque du diocèse unique de Corse. Il apparaît comme le commanditaire des tuiles de Sagone.

Le texte de l'estampille de type 1 semble faire référence à une demande très particulière. Le mot jubere marque un ordre et peut se traduire par "selon la volonté de Dieu»"19. Comment, dans ces conditions, ne pas penser à un songe durant lequel Dieu lui-même aurait intimé l'ordre à Paul, représentant de l'Église de Corse, de construire ou de réaménager à Sagone une basilique en l'honneur de saint Appien? Quoi qu'il en soit, son acte et les inscriptions indiquent qu'il a officiellement reconnu la sainteté d'Appien ${ }^{20}$.

La présence sur le site de deux types de timbres différents suscite toutefois des interrogations qui restent aujourd'hui sans réponse. Peut-être ne sont-ils pas strictement contemporains; on devrait alors imaginer deux campagnes de construction/reconstruction assez proches. Le timbre de type 2 pourrait dans ce cas être le plus récent : la démarche pourrait naturellement être impulsée par l'ordre venu de Dieu. Il est également possible que les tuiles aient été destinées à couvrir deux édifices différents: d'une part la basilique ellemême, de l'autre ses annexes. Le timbre de type 1 devrait alors être imprimé sur les tuiles utilisées pour couvrir l'édifice de culte.

\section{CONCLUSION}

Le réexamen archéologique de l'édifice de culte chrétien de Sagone antérieur à la cathédrale du XII ${ }^{\mathrm{e}}$ siècle renouvelle complètement la documentation et conduit à proposer des interprétations bien différentes de celles qui ont été formulées dans un premier temps par G. Moracchini-Mazel, puis par N. Duval et enfin par Ph. Pergola et F. Di Renzo.

L'hypothèse de la présence de deux édifices

18. Duperron 2019.

19. Cette interprétation nous a été suggérée par J. Guyon que nous remercions.

20. Sur cette question de reconnaissance des saints au $\mathrm{V}^{\mathrm{e}}$ siècle, voir en particulier Picard 1998, p. 341. 
d'époque paléochrétienne doit aujourd'hui être écartée. Il n'y a bien qu'une seule basilique dont les premières pierres sont posées vers les années 410-450. Cette chronologie est en accord avec les résultats fournis par l'étude de la cathédrale de Mariana, édifiée selon toutes vraisemblances au tout début du $\mathrm{V}^{\mathrm{e}}$ siècle. Ainsi, après la construction de cette basilique, l'édification de celle de Sagone pourrait être l'un des jalons de la structuration ecclésiastique de l'espace insulaire sous le contrôle de l'évêque de Corse. L'intervention de ce dernier est documentée par les estampilles sur tuiles, mais aussi peut-être par la typologie du presbyterium surélevé, fort ressemblant à celui de la cathédrale de référence. Il n'en reste pas moins que la basilique de Sagone, avec son abside à trois pans, présente une originalité dont on ne peut aujourd'hui expliquer les raisons. Plutôt qu'un lien avec les édifices de culte de Méditerranée orientale ${ }^{21}$, peut-être faut-il imaginer que la source d'inspiration a été l'architecture domestique, notamment celle des thermes dont le corpus désormais bien étoffé donne de nombreux exemples d'absides polygonales.

Enfin, l'élément nouveau le plus important est sans conteste la mise au jour de tuiles marquées d'estampilles dont le texte fait référence à saint Appien. Cette découverte invite d'une part à s'interroger sur cette pratique qui n'est certes pas propre à la Corse, mais qui est particulièrement bien documentée dans l'île ${ }^{22}$. Elle témoigne à la fois du contrôle par les évêques, ou du moins par le clergé, de la production de matériaux en terre cuite ainsi que de leur dynamisme en matière de construction.

Bien plus, ces estampilles remettent en question deux idées importantes généralement admises. Tout d'abord celle qui voudrait que le culte de tous "les saints vénérés en Corse au Moyen Âge et auxquels sont dédiées les églises les plus anciennes connues jusqu'ici" soit à mettre au crédit des évêques catholiques relégués dans l'île après la conférence tenue à Carthage en $484^{23}$. Or on sait désormais qu'à Sagone un culte était rendu à Appien au plus tard au milieu du $\mathrm{V}^{\mathrm{e}}$ siècle. Par ailleurs, on a considéré sur la base d'un document fort suspect du milieu du Moyen Âge que cet Appien était lui-même un évêque africain ${ }^{24}$. Pourtant, la découverte à proximité de la basilique de Sagone d'un mausolée de la première moitié du $\mathrm{V}^{\mathrm{e}}$ siècle vidé de sa sépulture quelques décennies plus tard, au moment même où l'on réorganise l'ensemble du presbyterium avec l'aménagement de passages latéraux et d'un autel coffre, amène aujourd'hui à reconsidérer cette question et à envisager une origine locale de ce personnage ${ }^{25}$.
21. Coroneo 2006, p.36-37.

22. Des tuiles avec estampilles chrétiennes des $\mathrm{V}^{\mathrm{e}}-\mathrm{VI}^{\mathrm{e}}$ siècles ont été découvertes à Sagone (4 types différents), mais aussi à Ajaccio (1 type), à Propriano (2 types), à PianottoliCaldarello ( 1 type), à Aleria ( 1 type) ainsi qu'à Mariana (2 types). Si ajoutent de nombreuses marques (croix, chrismes) tracées au doigt sur la pâte fraiche de tuiles utilisées (exclusivement?) pour la confection de tombes.
23. Pergola 2001, p. 22. Aucun élément ne permet de penser que des évêques africains ont été exilés en Corse avant cette date.

24. Moracchini-Mazel 2004.

25. Le mausolée et les transformations de la basilique feront l'objet d'un prochain article. 


\section{Bibliographie}

Coroneo 2006 = R. Coroneo, Chiese romaniche della Corsica, Cagliari, 2006.

Duperron $2019=$ G. Duperron (dir.), Vico (Corse-du-Sud), A Sullana - Sant'Appianu. Agglomération littorale et nécropole tardo-antiques, Rapport final d'opération, Ajaccio, 2019.

Duval 1995 = N. Duval, Sagone, dans N. Duval (dir.), Les premiers monuments chrétiens de la France, I. Sud-est et Corse, Paris, 1995, p. 324-329.

Istria 2011 = D. Istria, Étude architecturale de la cathédrale médiévale Sant'Appianu de Sagone, dans Archeologia dell'Architettura, XIV-2009, 2011, p. 63-74.

Istria $2014=\mathrm{D}$. Istria, Vico, dans F. Michel, D. Pasqualaggi (dir.), La Corse, Paris, 2014 (Carte archéologique de la Gaule), p. 136-141.

Istria - Françoise - Pellegrino 2012 = D. Istria avec la coll. de J. Françoise et E. Pellegrino, Les deux baptistères du groupe épiscopal de Sagone (Corse-du-Sud), dans Gallia, 69-2, 2012, p. 195-208.

Istria - Dixneuf - Françoise 2014 = D. Istria, D. Dixneuf, J. Françoise, Nouvelles données sur la chronologie du complexe paléochrétien de Mariana (Lucciana, Corse), dans Études corses, 79, 2014, p. 89-99.

Lanzoni 1927 = F. Lanzoni, Le diocesi d'Italia dale origini al principio del secolo VII (an.604), Faenza, 1927.

Moracchini-Mazel 1966 = G. Moracchini-Mazel, Une tuile estampillée découverte à Sagone (Corse), dans Bulletin de la
Société Nationale des Antiquaires de France, 1966, p. $167-$ 169.

Moracchini-Mazel 1972 = G. Moracchini-Mazel, La fouille des basiliques paléochrétiennes de Corse. Nouvelles découvertes, dans Actas del VIII Congresso Internacional de Arqueologia Cristiana, Barcelona, 1969, Barcelone-Cité du Vatican, 1972, p. 361-365.

Moracchini-Mazel 1988 = G. Moracchini-Mazel, Le premier baptistère de Sagone, dans Bulletin de la Société nationale des antiquaires de France, 1988, p. 388-391.

Moracchini-Mazel 2004 = G. Moracchini-Mazel, Corsica sacra, Porto Vecchio, 2004.

Pergola 2001 = Ph. Pergola, La Corse chrétienne dans l'Eglise universelle des origines à la fin du haut Moyen Age, dans Ph. Pergola, D. Istria (dir.), Corsica christiana, 2000 ans de christianisme, Catalogue de l'exposition du musée de la Corse à Corte, Ajaccio, 2001, p. 14-36.

Pergola - Di Renzo $2001=$ Ph. Pergola, F. Di Renzo, Cités et campagnes de Corse de la fin de l'Antiquité et du haut Moyen Age: évangélisation et christianisation, dans Ph. Pergola, D. Istria (dir.), Corsica christiana, 2000 ans de christianisme, Catalogue de l'exposition du musée de la Corse à Corte, Ajaccio, 2001, p. 106-124.

Picard $1998=$ J.-C. Picard, Le souvenir des évêques. Sépultures, listes épiscopale et culte des évêques en Italie du Nord des origines au Xe siècle, Rome, 1988 (Bibliothèque des Écoles françaises d'Athènes et de Rome, 268). 